Supporting Information

\title{
Programmed Design of Highly Crystalline Organic Semiconductor Patterns with Uniaxial Alignment via Blade Coating for High- Performance Organic Field-Effect Transistors
}

Kyunghun Kim, ${ }^{1,5}$ Kibeom Nam, ${ }^{2,5}$, Xinlin $\mathrm{Li}^{3}, 5$ Dong Yun Lee, ${ }^{2,}$, and Se Hyun Kim ${ }^{4, *}$

${ }^{1}$ Department of Chemical Engineering, Pohang University of Science and Technology, Pohang, 37673, Korea

${ }^{2}$ Department of Polymer Science and Engineering, Kyungpook National University, Daegu, 41566, Korea

${ }^{3}$ College of Electromechanical Engineering, Qingdao University, Qingdao 266071, China

${ }^{4}$ School of Engineering, Yeungnam University, 280 Daehak-Ro, Gyeongsan, Gyeongbuk, 38541 , Korea

${ }^{5}$ Contributed equally to this work.

*Corresponding Authors

E-mail: shkim97@yu.ac.kr (S. H. Kim)

E-mail: dongyunlee@knu.ac.kr (D. Y. Lee) 


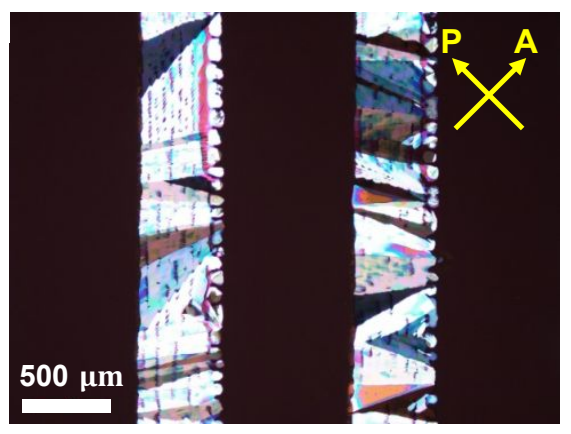

Figure S1. Polarized optical microscopy image of TIPS-PEN crystals printed at $0.05 \mathrm{~mm} / \mathrm{s}$. 

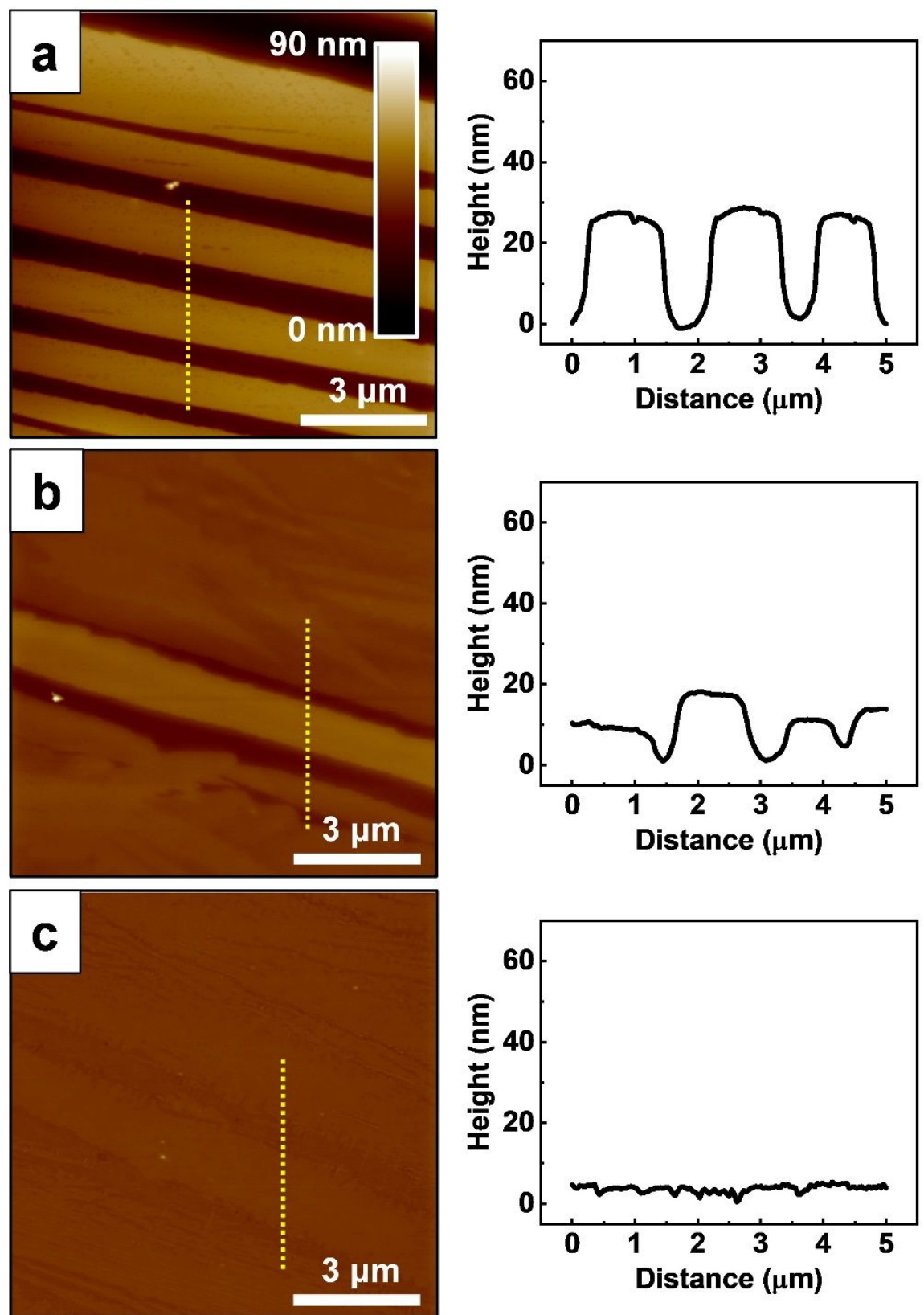

Figure S2. OM images of $\mathrm{SU}-8 / \mathrm{SiO}_{2}$ and $\mathrm{PUA} / \mathrm{SiO}_{2}$ substrates (left). pOM images of the substrates after blade coating the TIPS-PEN/PS solution (right). The axes of the polarizer (A) and analyzer $(\mathrm{A})$ in the microscope are indicated in the image. 


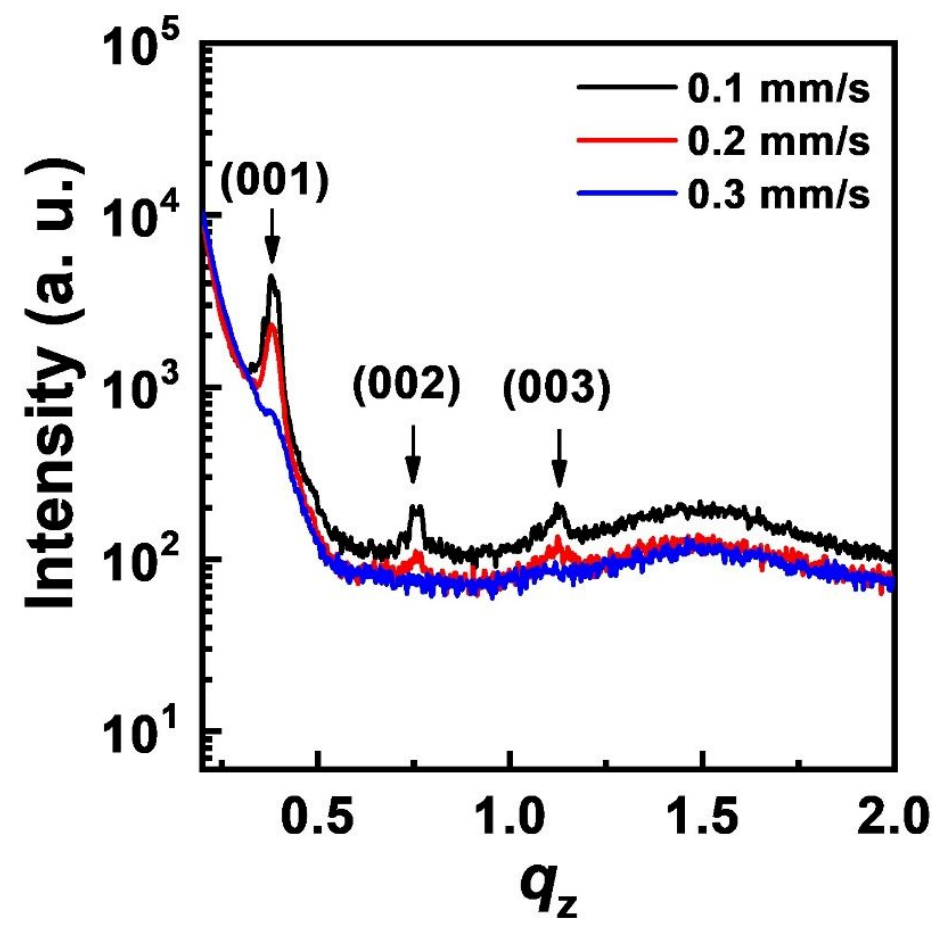

Figure S3. 1D X-ray diffraction profiles along the out-of-plane direction extracted from GIWAXS images with perpendicular beam incidences in Figure 4. 


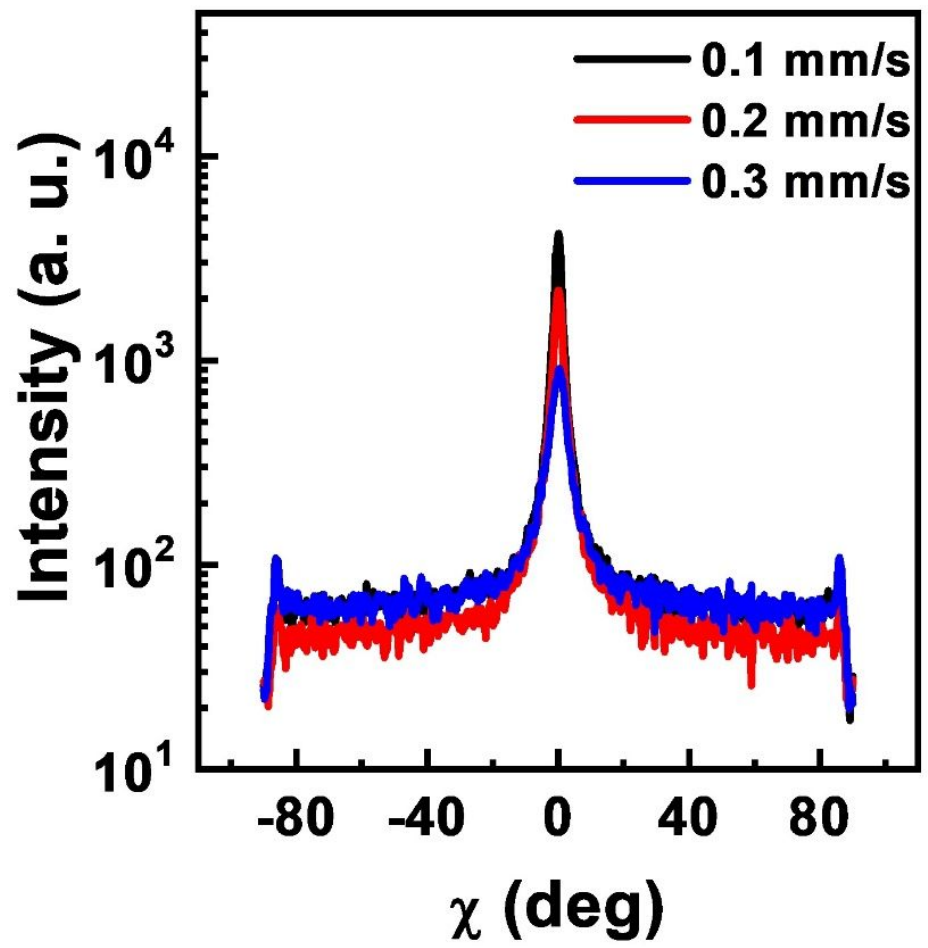

Figure S4. Pole figures plots of (001) peaks extracted from GIWAXS images with perpendicular beam incidences in Figure 4. The peak intensities were normalized by the volumes of crystals. 
(a)

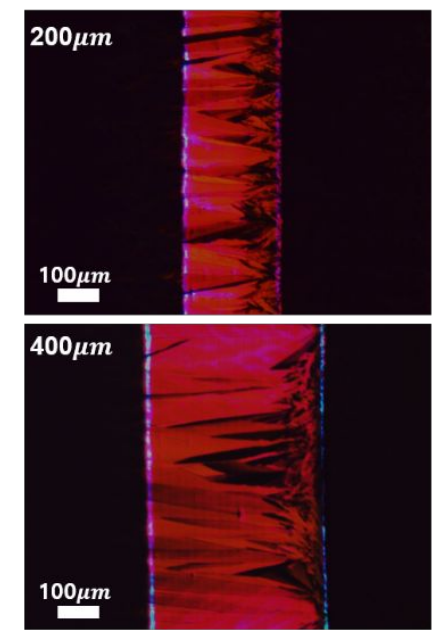

(b)

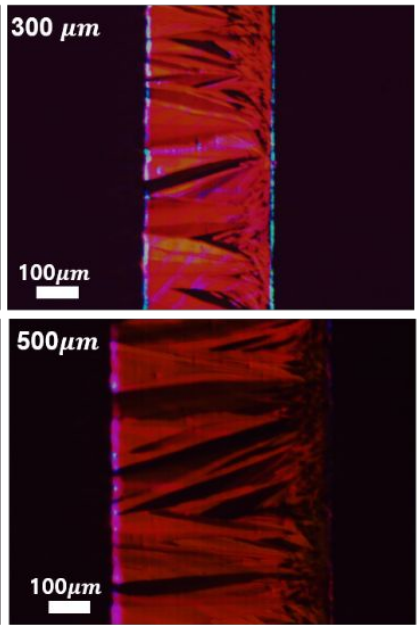

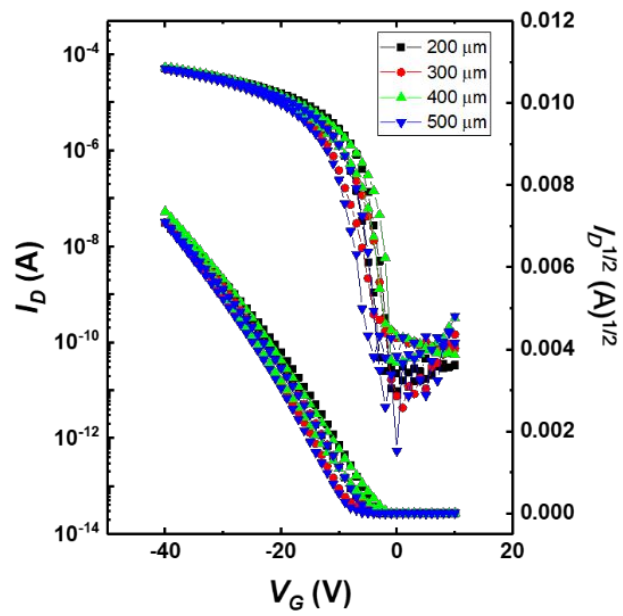

Figure S5. (a) pOM images of TIPS-PEN crystal patterns with various width of the crystal strip from $200 \mu \mathrm{m}$ to $500 \mu \mathrm{m}$ at the fixed printing speed of $0.1 \mathrm{~mm} / \mathrm{s}$ and (b) Transfer characteristics of the corresponding OFETs $\left(V_{\mathrm{D}}=-40 \mathrm{~V}\right)$ 

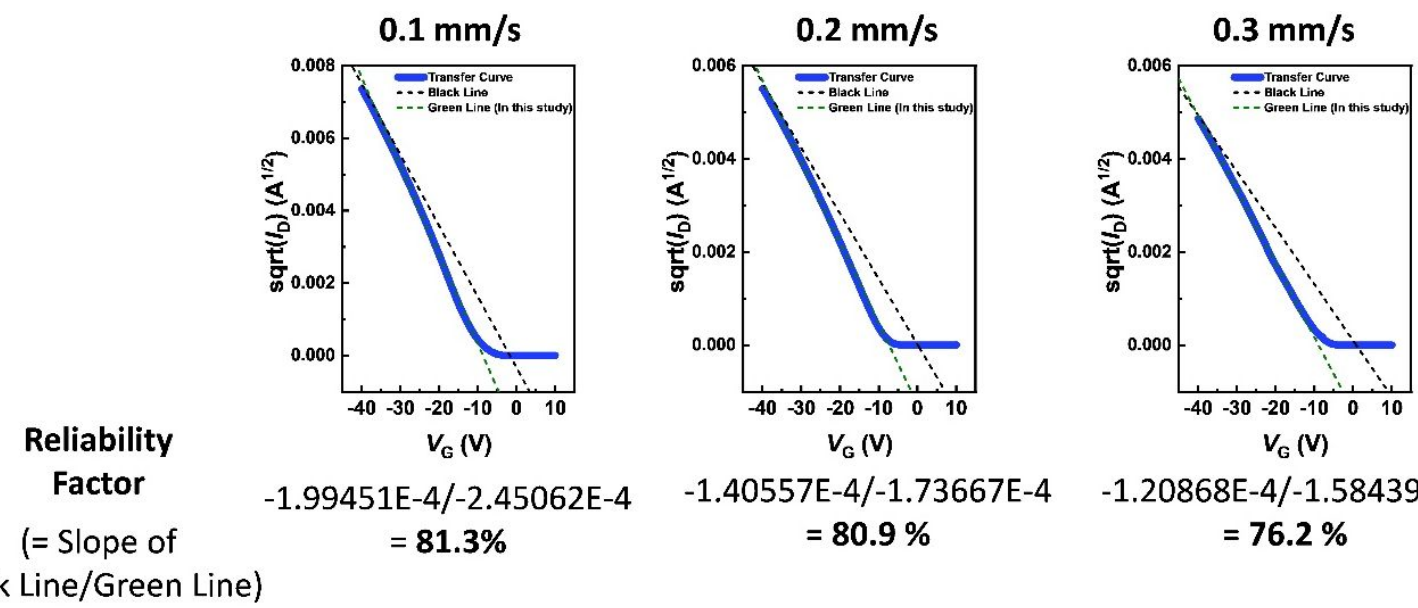

$-1.40557 \mathrm{E}-4 /-1.73667 \mathrm{E}-4$

$-1.20868 \mathrm{E}-4 /-1.58439 \mathrm{E}-4$ $=80.9 \%$

$=76.2 \%$ Black Line/Green Line)

Figure S6. Reliability factors of transfer curves in Figure $6 b$. 
Table S1. Crystallographic information of TIPS-PEN crystal patterns extracted from GIWAXS images in Figure 4. The coherence length was determined from the full width at half-maximum (FWHM) of (001) peaks in Figure S2.

\begin{tabular}{|c|c|c|}
\hline Shearing speeds & $\begin{array}{c}\text { Crystallographic } \\
\text { Parameters }\end{array}$ & Value \\
\hline \multirow{4}{*}{$0.1 \mathrm{~mm} / \mathrm{s}$} & $\mathrm{q}\left(\AA^{-1}\right)$ at $(001)$ plane & 0.380 \\
\cline { 2 - 3 } & d-spacing $(\AA)$ & 16.53 \\
\cline { 2 - 3 } & FWHM $\left(\AA^{-1}\right)$ & 0.03758 \\
\cline { 2 - 3 } & Coherence length $(\AA)$ & $\mathbf{1 6 7 . 2}$ \\
\hline \multirow{5}{*}{$0.2 \mathrm{~mm} / \mathrm{s}$} & $\mathrm{q}\left(\AA^{-1}\right)$ at $(001)$ plane & 0.380 \\
\cline { 2 - 3 } & d-spacing $(\AA)$ & 16.53 \\
\cline { 2 - 3 } & FWHM $\left(\AA^{-1}\right)$ & 0.04893 \\
\cline { 2 - 3 } & Coherence length $(\AA)$ & $\mathbf{1 2 8 . 4}$ \\
\hline \multirow{5}{*}{$0.3 \mathrm{~mm} / \mathrm{s}$} & $\mathrm{q}\left(\AA^{-1}\right)$ at $(001)$ plane & 0.380 \\
\cline { 2 - 3 } & d-spacing $(\AA)$ & 16.53 \\
\cline { 2 - 3 } & FWHM $\left(\AA^{-1}\right)$ & 0.33877 \\
\cline { 2 - 3 } & Coherence length $(\AA)$ & $\mathbf{1 8 . 5}$ \\
\hline
\end{tabular}

